\title{
Hilbert-type inequalities for time scale nabla calculus
}

\author{
H.M. Rezk ${ }^{1}$, Ghada AlNemer2* (D, H.A. Abd El-Hamid ${ }^{3}$, Abdel-Haleem Abdel-Aty ${ }^{4,5}$ (D),
}

Kottakkaran Sooppy Nisar ${ }^{6}$ and M. Zakarya ${ }^{7,8}$

${ }^{*}$ Correspondence:

gnnemer@pnu.edu.sa

${ }^{2}$ Department of Mathematical

Science, College of Science, Princess

Nourah bint Abdulrahman

University, P.O. Box 105862, Riyadh,

11656, Saudi Arabia

Full list of author information is

available at the end of the article

\begin{abstract}
This paper deals with the derivation of some new dynamic Hilbert-type inequalities in time scale nabla calculus. In proving the results, the basic idea is to use some algebraic inequalities, Hölder's inequality, and Jensen's time scale inequality. This generalization allows us not only to unify all the related results that exist in the literature on an arbitrary time scale, but also to obtain new outcomes that are analytical to the results of the delta time scale calculation.
\end{abstract}

MSC: Primary 26D10; secondary 26D15; 26E70

Keywords: Nabla calculus; Delta calculus; Hilbert's inequality; Time scales

\section{Introduction}

In recent years, Hilbert's dual-series inequality and its integral form [1, pp. 253-254] have been granted significant attention by many scholars (for example, see [2-10]). In particular, B. G. Pachpatte [11] established a new inequality close to that of Hilbert as follows. Let $k, r \geq 1, A_{s}=\sum_{m=1}^{s} a_{m} \geq 0$ and $B_{\vartheta}=\sum_{n=1}^{\vartheta} b_{n} \geq 0$. Then

$$
\begin{aligned}
\sum_{s=1}^{p} \sum_{\vartheta=1}^{q} \frac{A_{s}^{k} B_{\vartheta}^{r}}{s+\vartheta} \leq & C(k, r, p, q)\left(\sum_{s=1}^{p}(p-s+1)\left(A_{s}^{k-1} a_{s}\right)^{2}\right)^{\frac{1}{2}} \\
& \times\left(\sum_{\vartheta=1}^{q}(q-\vartheta+1)\left(B_{\vartheta}^{r-1} b_{\vartheta}\right)^{2}\right)^{\frac{1}{2}},
\end{aligned}
$$

where

$$
C(k, r, p, q)=\frac{1}{2} k r \sqrt{p q}
$$

In the same article [11], Pachpatte demonstrated the integral version of (1) as follows. Let $k, r \geq 1, \Pi(s)=\int_{0}^{s} \omega_{1}(\xi) d \xi \geq 0$ and $\Omega(\vartheta)=\int_{0}^{\vartheta} \omega_{2}(v) d v \geq 0$, for $s, \xi \in(0, x)$ and $\vartheta, v \in(0, y)$.

(c) The Author(s) 2020. This article is licensed under a Creative Commons Attribution 4.0 International License, which permits use, sharing, adaptation, distribution and reproduction in any medium or format, as long as you give appropriate credit to the original author(s) and the source, provide a link to the Creative Commons licence, and indicate if changes were made. The images or other third party material in this article are included in the article's Creative Commons licence, unless indicated otherwise in a credit line to the material. If material is not included in the article's Creative Commons licence and your intended use is not permitted by statutory regulation or exceeds the permitted use, you will need to obtain permission directly from the copyright holder. To view a copy of this licence, visit http://creativecommons.org/licenses/by/4.0/. 
Then

$$
\begin{aligned}
\int_{0}^{x} \int_{0}^{y} \frac{\Pi^{k}(s) \Omega^{r}(\vartheta)}{s+\vartheta} d s d \vartheta \leq & C^{*}(k, r, x, y)\left(\int_{0}^{x}(x-s)\left(\Pi^{k-1}(s) \omega_{1}(s)\right)^{2} d s\right)^{\frac{1}{2}} \\
& \times\left(\int_{0}^{y}(y-\vartheta)\left(\Omega^{r-1}(\vartheta) \omega_{2}(\vartheta)\right)^{2} d \vartheta\right)^{\frac{1}{2}}
\end{aligned}
$$

where

$$
C^{*}(k, r, x, y)=\frac{1}{2} k r \sqrt{x y}
$$

In [12], Young-Ho Kim gave some generalizations of (1) and (2) by introducing a parameter $\gamma>0$ as follows. Let $k, r \geq 1, A_{s}=\sum_{m=1}^{s} a_{m} \geq 0$ and $B_{\vartheta}=\sum_{n=1}^{\vartheta} b_{n} \geq 0$. Then

$$
\begin{aligned}
\sum_{s=1}^{p} \sum_{\vartheta=1}^{q} \frac{A_{s}^{k} B_{\vartheta}^{r}}{\left(s^{\gamma}+\vartheta \gamma\right)^{\frac{1}{\gamma}}} \leq & D(k, r, \gamma, p, q)\left(\sum_{s=1}^{p}(p-s+1)\left(A_{s}^{k-1} a_{s}\right)^{2}\right)^{\frac{1}{2}} \\
& \times\left(\sum_{\vartheta=1}^{q}(q-\vartheta+1)\left(B_{\vartheta}^{r-1} b_{\vartheta}\right)^{2}\right)^{\frac{1}{2}},
\end{aligned}
$$

where

$$
D(k, r, \gamma, p, q)=\left(\frac{1}{2}\right)^{\frac{1}{\gamma}} k r \sqrt{p q} .
$$

The integral version of (3) is established in the next consequence. Let $k, r \geq 1, \gamma>0, \Pi(s)=$ $\int_{0}^{s} \omega_{1}(\xi) d \xi \geq 0$, and $\Omega(\vartheta)=\int_{0}^{\vartheta} \omega_{2}(v) d v \geq 0$, for $s, \xi \in(0, x)$ and $\vartheta, v \in(0, y)$. Then

$$
\begin{aligned}
\int_{0}^{x} \int_{0}^{y} \frac{\Pi^{k}(s) \Omega^{r}(\vartheta)}{\left(s^{\gamma}+\vartheta \gamma\right)^{\frac{1}{\gamma}}} d s d \vartheta \leq & D^{*}(k, r, \gamma, x, y)\left(\int_{0}^{x}(x-s)\left(\Pi^{k-1}(s) \omega_{1}(s)\right)^{2} d s\right)^{\frac{1}{2}} \\
& \times\left(\int_{0}^{y}(y-\vartheta)\left(\Omega^{r-1}(\vartheta) \omega_{2}(\vartheta)\right)^{2} d \vartheta\right)^{\frac{1}{2}}
\end{aligned}
$$

where

$$
D^{*}(k, r, \gamma, x, y)=\left(\frac{1}{2}\right)^{\frac{1}{\gamma}} k r \sqrt{x y} .
$$

Another refinement of inequalities (1) and (2) has been made by Yang [13] as follows. Let $k, r \geq 1$ and $\lambda, \mu>1$ be constants such that $1 / \lambda+1 / \mu=1, A_{s}=\sum_{m=1}^{s} a_{m} \geq 0$, and $B_{\vartheta}=\sum_{n=1}^{\vartheta} b_{n} \geq 0$. Then

$$
\begin{aligned}
\sum_{s=1}^{p} \sum_{\vartheta=1}^{q} \frac{A_{s}^{k} B_{\vartheta}^{r}}{\mu s^{\frac{(\lambda-1)(\lambda+\mu)}{\lambda \mu}}+\lambda \vartheta^{\frac{(\mu-1)(\lambda+\mu)}{\lambda \mu}} \leq} & E(k, r, \lambda, \mu, p, q)\left(\sum_{s=1}^{p}(p-s+1)\left(A_{s}^{k-1} a_{s}\right)^{\lambda}\right)^{\frac{1}{\lambda}} \\
& \times\left(\sum_{\vartheta=1}^{q}(q-\vartheta+1)\left(B_{\vartheta}^{r-1} b_{\vartheta}\right)^{\mu}\right)^{\frac{1}{\mu}},
\end{aligned}
$$


where

$$
E(k, r, \lambda, \mu, p, q)=\frac{k r}{\lambda+\mu} p^{\frac{\lambda-1}{\lambda}} q^{\frac{\mu-1}{\mu}} .
$$

The integral version of (5) is established in the next consequence. Let $k, r \geq 1$ and $\lambda, \mu>1$ be constants such that $1 / \lambda+1 / \mu=1, \Pi(s)=\int_{0}^{s} \omega_{1}(\xi) d \xi \geq 0$, and $\Omega(\vartheta)=\int_{0}^{\vartheta} \omega_{2}(v) d v \geq 0$, for $s, \xi \in(0, x)$ and $\vartheta, v \in(0, y)$. Then

$$
\begin{aligned}
& \int_{0}^{x} \int_{0}^{y} \frac{\Pi^{k}(s) \Omega^{r}(\vartheta)}{\mu s^{\frac{(\lambda-1)(\lambda+\mu)}{\lambda \mu}}+\lambda \vartheta^{\frac{(\mu-1)(\lambda+\mu)}{\lambda \mu}}} d s d \vartheta \\
& \leq E^{*}(k, r, \lambda, \mu, x, y)\left(\int_{0}^{x}(x-s)\left(\Pi^{k-1}(s) \omega_{1}(s)\right)^{\lambda} d s\right)^{\frac{1}{\lambda}} \\
& \quad \times\left(\int_{0}^{y}(y-\vartheta)\left(\Omega^{r-1}(\vartheta) \omega_{2}(\vartheta)\right)^{\mu} d \vartheta\right)^{\frac{1}{\mu}},
\end{aligned}
$$

where

$$
E^{*}(k, r, \lambda, \mu, x, y)=\frac{k r}{\lambda+\mu} x^{\frac{\lambda-1}{\lambda}} y^{\frac{\mu-1}{\mu}}
$$

After construction of time scale calculus, dynamic inequalities have become the focus of interest, and classical inequalities have been established for any time scale $\mathbb{T}$. We can refer two surveys $[14,15]$ and a monograph [16] for exhibition of these results.

In [17] the researchers concluded some generalizations of inequalities (1) and (2) for time scale delta calculus. Specifically, they proved that if $s, \vartheta, \vartheta_{0} \in \mathbb{T}, \omega_{1}(s) \in$ $C_{r d}\left(\left[\vartheta_{0}, x\right)_{\mathbb{T}}, \mathbb{R}^{+}\right), \omega_{2}(\vartheta) \in C_{r d}\left(\left[\vartheta_{0}, y\right)_{\mathbb{T}}, \mathbb{R}^{+}\right), k, r \geq 1$ and $\lambda, \mu>1$ are constants such that $1 / \lambda+1 / \mu=1$, then for $s \in\left[\vartheta_{0}, x\right)_{\mathbb{T}}$ and $\vartheta \in\left[\vartheta_{0}, y\right)_{\mathbb{T}}$, one has

$$
\begin{aligned}
\int_{\vartheta_{0}}^{x} \int_{\vartheta_{0}}^{y} \frac{\Pi^{k}(s) \Omega^{r}(\vartheta)}{\mu\left(s-\vartheta_{0}\right)^{\frac{1}{\lambda}}+\lambda\left(\vartheta-\vartheta_{0}\right)^{\frac{1}{\mu}}} \Delta s \Delta \vartheta \\
\leq G(k, r, \lambda, \mu, x, y)\left(\int_{\vartheta_{0}}^{x}(\sigma(x)-s)\left(\Pi^{k-1}(\sigma(s)) \omega_{1}(s)\right)^{\lambda} \Delta s\right)^{\frac{1}{\lambda}} \\
\quad \times\left(\int_{\vartheta_{0}}^{y}(\sigma(y)-\vartheta)\left(\Omega^{r-1}(\sigma(\vartheta)) \omega_{2}(\vartheta)\right)^{\mu} \Delta \vartheta\right)^{\frac{1}{\mu}}
\end{aligned}
$$

where $\Pi(s)=\int_{\vartheta_{0}}^{s} \omega_{1}(\xi) \Delta \xi, \Omega(\vartheta)=\int_{\vartheta_{0}}^{\vartheta} \omega_{2}(\xi) \Delta \xi$, and

$$
G(k, r, \lambda, \mu, x, y)=\frac{k r}{\lambda \mu}\left(x-\vartheta_{0}\right)^{\frac{\lambda-1}{\lambda}}\left(y-\vartheta_{0}\right)^{\frac{\mu-1}{\mu}}
$$

Another refinement of (7) for time scale delta calculus has been made by Rezk et al. [18] as follows. Let $s, \vartheta, \vartheta_{0} \in \mathbb{T}, \omega_{1}(s) \in \mathrm{C}_{r d}\left(\left[\vartheta_{0}, x\right)_{\mathbb{T}}, \mathbb{R}^{+}\right), \omega_{2}(\vartheta) \in \mathrm{C}_{r d}\left(\left[\vartheta_{0}, y\right)_{\mathbb{T}}, \mathbb{R}^{+}\right), k, r \geq 1$ and $\lambda, \mu>1$ be constants such that $1 / \lambda+1 / \mu=1$, then for $s \in\left[\vartheta_{0}, \rho\right)_{\mathbb{T}}$ and $\vartheta \in\left[\vartheta_{0}, \tau\right)_{\mathbb{T}}$, one 
has

$$
\begin{aligned}
& \int_{\vartheta_{0}}^{x} \int_{\vartheta_{0}}^{y} \frac{\Pi^{k}(s) \Omega^{r}(\vartheta)}{\mu\left(s-\vartheta_{0}\right)^{\frac{(\lambda-1)(\lambda+\mu)}{\lambda \mu}}+\lambda\left(\vartheta-\vartheta_{0}\right)^{\frac{(\mu-1)(\lambda+\mu)}{\lambda \mu}}} \Delta s \Delta \vartheta \\
& \leq G^{*}(k, r, \lambda, \mu, x, y)\left(\int_{\vartheta_{0}}^{x}(\sigma(x)-s)\left(\Pi^{k-1}(\sigma(s)) \omega_{1}(s)\right)^{\lambda} \Delta s\right)^{\frac{1}{\lambda}} \\
& \quad \times\left(\int_{\vartheta_{0}}^{y}(\sigma(y)-\vartheta)\left(\Omega^{r-1}(\sigma(\vartheta)) \omega_{2}(\vartheta)\right)^{\mu} \Delta \vartheta\right)^{\frac{1}{\mu}}
\end{aligned}
$$

where $\Pi(s)=\int_{\vartheta_{0}}^{s} \omega_{1}(\xi) \Delta \xi, \Omega(\vartheta)=\int_{\vartheta_{0}}^{\vartheta} \omega_{2}(\xi) \Delta \xi$, and

$$
G^{*}(k, r, \lambda, \mu, x, y)=\frac{k r}{\lambda+\mu}\left(x-\vartheta_{0}\right)^{\frac{\lambda-1}{\lambda}}\left(y-\vartheta_{0}\right)^{\frac{\mu-1}{\mu}} .
$$

For developing of Hilbert's inequalities for time scale delta calculus, we refer the reader to the articles [19-29]. Although there are many results for time scale calculus in the sense of delta derivative, there is not much done for the nabla derivative. Therefore the major contribution of this article is to extend Hilbert-type inequalities for the nabla time scale calculus and to unify them for an arbitrary time scale. The main theorems are inspired from the paper [18] which presents the corresponding results for time scale delta calculus. By obtaining their nabla versions, we can show the generalizations of these inequalities for different types of time scales $\mathbb{T}$, such as real numbers and integers.

The structure of this paper can be listed as follows. Section 2 presents the fundamental concepts of the time scale calculus in terms of delta and nabla derivatives. Section 3 is devoted to main results, which are to generalize inequalities (5) and (6) for the nabla time scale calculus and so, to obtain nabla calculus versions of (9) and several inequalities of Hilbert's type in [18].

\section{Preliminaries}

In this section, the fundamental theories of the time scale delta and nabla calculi will be presented. Time scale calculus whose detailed information can be found in [30,31] has been invented in order to unify continuous and discrete analysis.

A nonempty closed subset of $\mathbb{R}$ is named a time scale and is denoted by $\mathbb{T}$. For $\vartheta \in \mathbb{T}$, if $\inf \emptyset=\sup \mathbb{T}$ and $\sup \emptyset=\inf \mathbb{T}$, then the forward jump operator $\sigma: \mathbb{T} \rightarrow \mathbb{T}$ and the backward jump operator $\rho: \mathbb{T} \rightarrow \mathbb{T}$ are defined as $\sigma(\vartheta)=\inf (\vartheta, \infty)_{\mathbb{T}}$ and $\rho(\vartheta)=\sup (-\infty, \vartheta)_{\mathbb{T}}$, respectively. From the above two concepts, it can be mentioned that a point $\vartheta \in \mathbb{T}$ with inf $\mathbb{T}<\vartheta<\sup \mathbb{T}$ is named right-scattered if $\sigma(\vartheta)>\vartheta$, right-dense if $\sigma(\vartheta)=\vartheta$, leftscattered if $\rho(\vartheta)<\vartheta$ and left-dense if $\rho(\vartheta)=\vartheta$.

The $\Delta$-derivative of $\psi: \mathbb{T} \rightarrow \mathbb{R}$ at $\vartheta \in \mathbb{T}^{k}=\mathbb{T} /(\rho(\sup \mathbb{T})$, sup $\mathbb{T}]$ denoted by $\psi^{\Delta}(\vartheta)$ is the number enjoying the property that for all $\varepsilon>0$ there is a neighborhood $U$ of $\vartheta \in \mathbb{T}^{k}$ such that

$$
\left|\psi(\sigma(\vartheta))-\psi(s)-\psi^{\Delta}(\vartheta)(\sigma(\vartheta)-s)\right| \leq \varepsilon|\sigma(\vartheta)-s|, \quad \text { for all } s \in U
$$

The $\nabla$-derivative of $\psi: \mathbb{T} \rightarrow \mathbb{R}$ at $\vartheta \in \mathbb{T}_{k}=\mathbb{T} /[\inf \mathbb{T}, \sigma(\inf \mathbb{T}))$ denoted by $\psi^{\nabla}(\xi)$ is the number enjoying the property that for all $\varepsilon>0$ there is a neighborhood $V$ of $\vartheta \in \mathbb{T}_{k}$ such 
that

$$
\left|\psi(\vartheta)-\psi(\rho(s))-\psi^{\nabla}(\vartheta)(\vartheta-\rho(s))\right| \leq \varepsilon|s-\rho(\vartheta)|, \quad \text { for all } s \in V
$$

A function $\psi: \mathbb{T} \rightarrow \mathbb{R}$ is $r d$-continuous if it is continuous at each right-dense point in $\mathbb{T}$ and $\lim _{s \rightarrow \vartheta^{-}} \psi(s)$ exists as a finite number for all left-dense points in $\mathbb{T}$. The set $C_{r d}(\mathbb{T}, \mathbb{R})$ represents the class of real, $r d$-continuous functions defined on $\mathbb{T}$. If $\psi \in C_{r d}(\mathbb{T}, \mathbb{R})$, then there exists a function $\Psi(\vartheta)$ such that $\Psi^{\Delta}(\vartheta)=\psi(\vartheta)$ and the delta integral of $\psi$ is defined by

$$
\int_{x_{0}}^{x} \psi(\vartheta) \Delta \vartheta=\Psi(x)-\Psi\left(x_{0}\right)
$$

A function $\psi: \mathbb{T} \rightarrow \mathbb{R}$ is $l d$-continuous if it is continuous at each left-dense point in $\mathbb{T}$ and $\lim _{s \rightarrow \vartheta^{+}} \psi(s)$ exists as a finite number for all right-dense points in $\mathbb{T}$. The set $C_{l d}(\mathbb{T}, \mathbb{R})$ represents the class of real, $l d$-continuous functions defined on $\mathbb{T}$. If $\psi \in C_{l d}(\mathbb{T}, \mathbb{R})$, then there exists a function $\Psi(\vartheta)$ such that $\Psi^{\nabla}(\vartheta)=\psi(\vartheta)$ and the nabla integral of $\psi$ is defined by

$$
\int_{x_{0}}^{x} \psi(\vartheta) \nabla \vartheta=\Psi(x)-\Psi\left(x_{0}\right)
$$

In the following, we display some basic lemmas and algebraic inequalities that play a key role in proving the major findings of this paper.

Lemma 2.1 (Nabla Hölder's Inequality [32]) Let $x_{0}, x \in \mathbb{T}$. For $\xi, \psi \in \mathrm{C}_{l d}\left(\left[x_{0}, x\right]_{\mathbb{T}}, \mathbb{R}\right)$, we have

$$
\int_{x_{0}}^{x} \xi(\vartheta) \psi(\vartheta) \nabla \vartheta \leq\left(\int_{x_{0}}^{x} \xi^{\lambda}(\vartheta) \nabla \vartheta\right)^{\frac{1}{\lambda}}\left(\int_{x_{0}}^{x} \psi^{\mu}(\vartheta) \nabla \vartheta\right)^{\frac{1}{\mu}}
$$

where $\lambda, \mu>1$ with $1 / \lambda+1 / \mu=1$.

Lemma 2.2 (Nabla Jensen's Inequality [33, Theorem 3.4]) Let $x_{0}, x \in \mathbb{T}$ and $m, n \in \mathbb{R}$. Assume that $\xi \in \mathrm{C}_{l d}\left(\left[x_{0}, x\right]_{\mathbb{T}},(m, n)\right)$ and $\psi \in \mathrm{C}_{l d}\left(\left[x_{0}, x\right]_{\mathbb{T}}, \mathbb{R}\right)$ are nonnegative with $\int_{x_{0}}^{x} \xi(\eta) \Delta \eta>0$. If $\Theta \in \mathrm{C}((m, n), \mathbb{R})$ is a convex function, then

$$
\Theta\left(\frac{\int_{x_{0}}^{x} \xi(\eta) \psi(\eta) \nabla \eta}{\int_{x_{0}}^{x} \xi(\eta) \nabla \eta}\right) \leq \frac{\int_{x_{0}}^{x} \xi(\eta) \Theta(\psi(\eta)) \nabla \eta}{\int_{x_{0}}^{x} \xi(\eta) \nabla \eta} .
$$

Lemma 2.3 (The power rule for nabla derivative [33, Lemma 3.1]) Let $x_{0}, x \in \mathbb{T}, \psi \in$ $\mathrm{C}_{l d}\left(\left[x_{0}, x\right]_{\mathbb{T}}, \mathbb{R}\right)$ be a nonnegative function, and $\gamma \geq 1$ a real constant. Then

$$
\left(\int_{x_{0}}^{x} \psi(\xi) \nabla \xi\right)^{\gamma} \leq \gamma \int_{x_{0}}^{x} \psi(v)\left(\int_{a}^{v} \psi(\xi) \nabla \xi\right)^{\gamma-1} \nabla v .
$$


Lemma 2.4 (Young's inequality [34]) Let $\delta>0, \Lambda_{q}>0$ and $\sum_{q=1}^{n} \Lambda_{q}=\Upsilon_{n}$. Then

$$
\left\{\prod_{q=1}^{n} s_{q}^{\Lambda_{q}}\right\}^{\frac{1}{\Upsilon_{n}}} \leq\left\{\frac{1}{\Upsilon_{n}} \sum_{q=1}^{n} \Lambda_{q} s_{q}^{\delta}\right\}^{\frac{1}{\delta}}
$$

Lemma 2.5 ([33, Lemma 3.2]) Let $s, \vartheta, \vartheta_{0} \in \mathbb{T}$ with $s, \vartheta \geq \vartheta_{0}$ and $\psi \in \mathrm{C}_{l d}\left([a, b]_{\mathbb{T}}, \mathbb{R}\right)$. Then

$$
\int_{\vartheta_{0}}^{s}\left(\int_{\vartheta_{0}}^{\vartheta} \psi(\xi) \nabla \xi\right) \nabla \vartheta=\int_{\vartheta_{0}}^{s}\left(\int_{\rho(\xi)}^{s} \psi(\xi) \nabla s\right) \nabla \xi=\int_{\vartheta_{0}}^{s}(s-\rho(\xi)) \psi(\xi) \nabla \xi .
$$

\section{Key results}

In this section, we focus on obtaining the corresponding outcomes for the nabla time scale calculation in [18]. We must assume that all functions found in the theorem statements are nonnegative, ld-continuous, $\nabla$-differentiable, and locally nabla integrable.

Theorem 3.1 Let $s, \vartheta, \vartheta_{0} \in \mathbb{T}$ and $\omega_{1} \in \mathrm{C}_{l d}\left(\left[\vartheta_{0}, x\right]_{\mathbb{T}}, \mathbb{R}^{+}\right), \omega_{2} \in \mathrm{C}_{l d}\left(\left[\vartheta_{0}, y\right]_{\mathbb{T}}, \mathbb{R}^{+}\right)$. Define

$$
\Pi(s)=\int_{\vartheta_{0}}^{s} \omega_{1}(\xi) \nabla \xi \quad \text { and } \quad \Omega(\vartheta)=\int_{\vartheta_{0}}^{\vartheta} \omega_{2}(\xi) \nabla \xi
$$

Then for $s \in\left[\vartheta_{0}, x\right]_{\mathbb{T}}$ and $\vartheta \in\left[\vartheta_{0}, y\right]_{\mathbb{T}}$, we have

$$
\begin{gathered}
\int_{\vartheta_{0}}^{x} \int_{\vartheta_{0}}^{y} \frac{\Pi^{k}(s) \Omega^{r}(\vartheta)}{\mu\left(s-\vartheta_{0}\right)^{\frac{(\lambda-1)(\lambda+\mu)}{\lambda \mu}}+\lambda\left(\vartheta-\vartheta_{0}\right)^{\frac{(\mu-1)(\lambda+\mu)}{\lambda \mu}}} \nabla s \nabla \vartheta \\
\leq H(k, r, \lambda, \mu, x, y)\left(\int_{\vartheta_{0}}^{x}(x-\rho(s))\left(\Pi^{k-1}(s) \omega_{1}(s)\right)^{\lambda} \nabla s\right)^{\frac{1}{\lambda}} \\
\quad \times\left(\int_{\vartheta_{0}}^{y}(y-\rho(\vartheta))\left(\Omega^{r-1}(\vartheta) \omega_{2}(\vartheta)\right)^{\mu} \nabla \vartheta\right)^{\frac{1}{\mu}}
\end{gathered}
$$

where

$$
H(k, r, \lambda, \mu, x, y)=\frac{k r}{\lambda+\mu}\left(x-\vartheta_{0}\right)^{\frac{\lambda-1}{\lambda}}\left(y-\vartheta_{0}\right)^{\frac{\mu-1}{\mu}} .
$$

Proof By using (13), we obtain

$$
\Pi^{k}(s) \leq k \int_{\vartheta_{0}}^{s} \Pi^{k-1}(\xi) \omega_{1}(\xi) \nabla \xi
$$

and

$$
\Omega^{r}(\vartheta) \leq r \int_{\vartheta_{0}}^{\vartheta} \Omega^{r-1}(\xi) \omega_{2}(\xi) \nabla \xi
$$

Then, we have

$$
\Pi^{k}(s) \Omega^{r}(\vartheta) \leq k r\left(\int_{\vartheta_{0}}^{s} \Pi^{k-1}(\xi) \omega_{1}(\xi) \nabla \xi\right)\left(\int_{\vartheta_{0}}^{\vartheta} \Omega^{r-1}(\xi) \omega_{2}(\xi) \nabla \xi\right) .
$$


Applying (11) to $\int_{\vartheta_{0}}^{s} \Pi^{k-1}(\xi) \omega_{1}(\xi) \nabla \xi$ with indices $\lambda$ and $\lambda /(\lambda-1)$, we find that

$$
\int_{\vartheta_{0}}^{s} \Pi^{k-1}(\xi) \omega_{1}(\xi) \nabla \xi \leq\left(s-\vartheta_{0}\right)^{\frac{\lambda-1}{\lambda}}\left(\int_{\vartheta_{0}}^{s}\left(\Pi^{k-1}(\xi) \omega_{1}(\xi)\right)^{\lambda} \nabla \xi\right)^{\frac{1}{\lambda}}
$$

while doing the same to the integral $\int_{\vartheta_{0}}^{\vartheta} \Omega^{r-1}(\xi) \omega_{2}(\xi) \nabla \xi$ with indices $\mu$ and $\mu /(\mu-1)$, we find that

$$
\int_{\vartheta_{0}}^{\vartheta} \Omega^{r-1}(\xi) \omega_{2}(\xi) \nabla \xi \leq\left(\vartheta-\vartheta_{0}\right)^{\frac{\mu-1}{\mu}}\left(\int_{\vartheta_{0}}^{\vartheta}\left(\Omega^{r-1}(\xi) \omega_{2}(\xi)\right)^{\mu} \nabla \xi\right)^{\frac{1}{\mu}} .
$$

From (20), (21), and (22), we get

$$
\begin{aligned}
\Pi^{k}(s) \Omega^{r}(\vartheta) \leq & k r\left(s-\vartheta_{0}\right)^{\frac{\lambda-1}{\lambda}}\left(\vartheta-\vartheta_{0}\right)^{\frac{\mu-1}{\mu}}\left(\int_{\vartheta_{0}}^{s}\left(\Pi^{k-1}(\xi) \omega_{1}(\xi)\right)^{\lambda} \nabla \xi\right)^{\frac{1}{\lambda}} \\
& \times\left(\int_{\vartheta_{0}}^{\vartheta}\left(\Omega^{r-1}(\xi) \omega_{2}(\xi)\right)^{\mu} \nabla \xi\right)^{\frac{1}{\mu}} .
\end{aligned}
$$

Using inequality (14), we note

$$
\left(s_{1}^{\Lambda_{1}} s_{2}^{\Lambda_{2}}\right)^{\frac{\delta}{\Lambda_{1}+\Lambda_{2}}} \leq \frac{1}{\Lambda_{1}+\Lambda_{2}}\left(\Lambda_{1} s_{1}^{\delta}+\Lambda_{2} s_{2}^{\delta}\right)
$$

Now, by setting $s_{1}=\left(s-\vartheta_{0}\right)^{\lambda-1}, s_{2}=\left(\vartheta-\vartheta_{0}\right)^{\mu-1}, \Lambda_{1}=1 / \lambda, \Lambda_{1}=1 / \mu$, and $\delta=\Lambda_{1}+\Lambda_{2}$ in (24), we get

$$
\left(s-\vartheta_{0}\right)^{\frac{\lambda-1}{\lambda}}\left(\vartheta-\vartheta_{0}\right)^{\frac{\mu-1}{\mu}} \leq \frac{\lambda \mu}{\lambda+\mu}\left(\frac{\left(s-\vartheta_{0}\right)^{\frac{(\lambda-1)(\lambda+\mu)}{\lambda \mu}}}{\lambda}+\frac{\left(\vartheta-\vartheta_{0}\right)^{\frac{(\mu-1)(\lambda+\mu)}{\lambda \mu}}}{\mu}\right) .
$$

Substituting (25) into (23) yields

$$
\begin{aligned}
\Pi^{k}(s) \Omega^{r}(\vartheta) \leq & \frac{k r \lambda \mu}{\lambda+\mu}\left(\frac{\left(s-\vartheta_{0}\right)^{\frac{(\lambda-1)(\lambda+\mu)}{\lambda \mu}}}{\lambda}+\frac{\left(\vartheta-\vartheta_{0}\right)^{\frac{(\mu-1)(\lambda+\mu)}{\lambda \mu}}}{\mu}\right) \\
& \times\left(\int_{\vartheta_{0}}^{s}\left(\Pi^{k-1}(\xi) \omega_{1}(\xi)\right)^{\lambda} \nabla \xi\right)^{\frac{1}{\lambda}}\left(\int_{\vartheta_{0}}^{\vartheta}\left(\Omega^{r-1}(\xi) \omega_{2}(\xi)\right)^{\mu} \nabla \xi\right)^{\frac{1}{\mu}} .
\end{aligned}
$$

Dividing both sides of $(26)$ by $\mu\left(s-\vartheta_{0}\right)^{[(\lambda-1)(\lambda+\mu)] / \lambda \mu}+\lambda\left(\vartheta-\vartheta_{0}\right)^{[(\mu-1)(\lambda+\mu)] / \lambda \mu}$, we obtain

$$
\begin{gathered}
\frac{\Pi^{k}(s) \Omega^{r}(\vartheta)}{\mu\left(s-\vartheta_{0}\right)^{\frac{(\lambda-1)(\lambda+\mu)}{\lambda \mu}}+\lambda\left(\vartheta-\vartheta_{0}\right)^{\frac{(\mu-1)(\lambda+\mu)}{\lambda \mu}}} \\
\leq \frac{k r}{\lambda+\mu}\left(\int_{\vartheta_{0}}^{s}\left(\Pi^{k-1}(\xi) \omega_{1}(\xi)\right)^{\lambda} \nabla \xi\right)^{\frac{1}{\lambda}} \\
\times\left(\int_{\vartheta_{0}}^{\vartheta}\left(\Omega^{r-1}(\xi) \omega_{2}(\xi)\right)^{\mu} \nabla \xi\right)^{\frac{1}{\mu}} .
\end{gathered}
$$


Integrating both sides of (27) and using (11) again, we get

$$
\begin{gathered}
\int_{\vartheta_{0}}^{x} \int_{\vartheta_{0}}^{y} \frac{\Pi^{k}(s) \Omega^{r}(\vartheta)}{\mu\left(s-\vartheta_{0}\right)^{\frac{(\lambda-1)(\lambda+\mu)}{\lambda \mu}}+\lambda\left(\vartheta-\vartheta_{0}\right)^{\frac{(\mu-1)(\lambda+\mu)}{\lambda \mu}}} \nabla s \nabla \vartheta \\
\leq \frac{k r}{\lambda+\mu}\left(x-\vartheta_{0}\right)^{\frac{\lambda-1}{\lambda}}\left(y-\vartheta_{0}\right)^{\frac{\mu-1}{\mu}} \\
\quad \times\left(\int_{\vartheta_{0}}^{x}\left(\int_{\vartheta_{0}}^{s}\left(\Pi^{k-1}(\xi) \omega_{1}(\xi)\right)^{\lambda} \nabla \xi\right) \nabla s\right)^{\frac{1}{\lambda}} \\
\quad \times\left(\int_{\vartheta_{0}}^{y}\left(\int_{\vartheta_{0}}^{\vartheta}\left(\Omega^{r-1}(\xi) \omega_{2}(\xi)\right)^{\mu} \nabla \xi\right) \nabla \vartheta\right)^{\frac{1}{\mu}} .
\end{gathered}
$$

Applying Lemma 2.5 on (28), we conclude that

$$
\begin{aligned}
& \int_{\vartheta_{0}}^{x} \int_{\vartheta_{0}}^{y} \frac{\Pi^{k}(s) \Omega^{r}(\vartheta)}{\mu\left(s-\vartheta_{0}\right)^{\frac{(\lambda-1)(\lambda+\mu)}{\lambda \mu}}+\lambda\left(\vartheta-\vartheta_{0}\right)^{\frac{(\mu-1)(\lambda+\mu)}{\lambda \mu}} \nabla s \nabla \vartheta} \\
& \leq \frac{k r}{\lambda+\mu}\left(x-\vartheta_{0}\right)^{\frac{\lambda-1}{\lambda}}\left(y-\vartheta_{0}\right)^{\frac{\mu-1}{\mu}}\left(\int_{\vartheta_{0}}^{x}(x-\rho(s))\left(\Pi^{k-1}(s) \omega_{1}(s)\right)^{\lambda} \nabla s\right)^{\frac{1}{\lambda}} \\
& \quad \times\left(\int_{\vartheta_{0}}^{y}(y-\rho(\vartheta))\left(\Omega^{r-1}(\vartheta) \omega_{2}(\vartheta)\right)^{\mu} \nabla \vartheta\right)^{\frac{1}{\mu}} \\
& =H(k, r, \lambda, \mu, x, y)\left(\int_{\vartheta_{0}}^{x}(x-\rho(s))\left(\Pi^{k-1}(s) \omega_{1}(s)\right)^{\lambda} \nabla s\right)^{\frac{1}{\lambda}} \\
& \quad \times\left(\int_{\vartheta_{0}}^{y}(y-\rho(\vartheta))\left(\Omega^{r-1}(\vartheta) \omega_{2}(\vartheta)\right)^{\mu} \nabla \vartheta\right)^{\frac{1}{\mu}}
\end{aligned}
$$

that is, (17) is true.

Remark 3.2 By setting $1 / \lambda+1 / \mu=1$ in (24), we obtain

$$
\left(s_{1}^{\Lambda_{1}} s_{2}^{\Lambda_{2}}\right) \leq \frac{1}{\Lambda_{1}+\Lambda_{2}}\left(\Lambda_{1} s_{1}^{\Lambda_{1}+\Lambda_{2}}+\Lambda_{2} s_{2}^{\Lambda_{1}+\Lambda_{2}}\right) .
$$

Hence, by applying (29) on the right-hand side of (17) in Theorem 3.1, we get

$$
\begin{aligned}
\int_{\vartheta_{0}}^{x} \int_{\vartheta_{0}}^{y} \frac{\Pi^{k}(s) \Omega^{r}(\vartheta)}{\mu\left(s-\vartheta_{0}\right)^{\frac{(\lambda-1)(\lambda+\mu)}{\lambda \mu}}+\lambda\left(\vartheta-\vartheta_{0}\right)^{\frac{(\mu-1)(\lambda+\mu)}{\lambda \mu}} \nabla s \nabla \vartheta} \\
\leq \frac{\lambda \mu k r}{(\lambda+\mu)^{2}}\left(x-\vartheta_{0}\right)^{\frac{\lambda-1}{\lambda}}\left(y-\vartheta_{0}\right)^{\frac{\mu-1}{\mu}} \\
\quad \times\left\{\frac{1}{\lambda}\left(\int_{\vartheta_{0}}^{x}(x-\rho(s))\left(\Pi^{k-1}(s) \omega_{1}(s)\right)^{\lambda} \nabla s\right)^{\frac{\lambda+\mu}{\lambda \mu}}\right. \\
\left.+\frac{1}{\mu}\left(\int_{\vartheta_{0}}^{y}(y-\rho(\vartheta))\left(\Omega^{r-1}(\vartheta) \omega_{2}(\vartheta)\right)^{\mu} \nabla \vartheta\right)^{\frac{\lambda+\mu}{\lambda \mu}}\right\} .
\end{aligned}
$$


Corollary 3.1 If we take $1 / \lambda+1 / \mu=1$ in (17), then

$$
\begin{aligned}
& \int_{\vartheta_{0}}^{x} \int_{\vartheta_{0}}^{y} \frac{\Pi^{k}(s) \Omega^{r}(\vartheta)}{\mu\left(s-\vartheta_{0}\right)^{\lambda-1}+\lambda\left(\vartheta-\vartheta_{0}\right)^{\mu-1}} \nabla s \nabla \vartheta \\
& \leq H^{*}(k, r, \lambda, \mu, x, y)\left(\int_{\vartheta_{0}}^{x}(x-\rho(s))\left(\Pi^{k-1}(s) \omega_{1}(s)\right)^{\lambda} \nabla s\right)^{\frac{1}{\lambda}} \\
& \quad \times\left(\int_{\vartheta_{0}}^{y}(y-\rho(\vartheta))\left(\Omega^{r-1}(\vartheta) \omega_{2}(\vartheta)\right)^{\mu} \nabla \vartheta\right)^{\frac{1}{\mu}}
\end{aligned}
$$

where

$$
H^{*}(k, r, \lambda, \mu, x, y)=\frac{k r}{\lambda \mu}\left(x-\vartheta_{0}\right)^{\frac{\lambda-1}{\lambda}}\left(y-\vartheta_{0}\right)^{\frac{\mu-1}{\mu}} .
$$

Remark 3.3 As a particular case of Corollary 3.1, if $\lambda=\mu=2$, then we have

$$
\begin{aligned}
\int_{\vartheta_{0}}^{x} \int_{\vartheta_{0}}^{y} \frac{\Pi^{k}(s) \Omega^{r}(\vartheta)}{s+\vartheta-2 \vartheta_{0}} \nabla s \nabla \vartheta \\
\leq \frac{1}{2} k r\left(\left(x-\vartheta_{0}\right) \int_{\vartheta_{0}}^{x}(x-\rho(s))\left(\Pi^{k-1}(s) \omega_{1}(s)\right)^{2} \nabla s\right)^{\frac{1}{2}} \\
\quad \times\left(\left(y-\vartheta_{0}\right) \int_{\vartheta_{0}}^{y}(y-\rho(\vartheta))\left(\Omega^{r-1}(\vartheta) \omega_{2}(\vartheta)\right)^{2} \nabla \vartheta\right)^{\frac{1}{2}}
\end{aligned}
$$

which is [33, Theorem 3.3].

Remark 3.4 Clearly, for $\mathbb{T}=\mathbb{Z}$ or $\mathbb{T}=\mathbb{R}$, and $\vartheta_{0}=0$, together with $\rho(u)=u-1$ or $\rho(u)=u$, (17) reduces to (5) or (6), respectively.

Remark 3.5 In Theorem 3.1, if we take $k=r=1$, then we have

$$
\begin{gathered}
\int_{\vartheta_{0}}^{x} \int_{\vartheta_{0}}^{y} \frac{\Pi(s) \Omega(\vartheta)}{\mu\left(s-\vartheta_{0}\right)^{\frac{(\lambda-1)(\lambda+\mu)}{\lambda \mu}}+\lambda\left(\vartheta-\vartheta_{0}\right)^{\frac{(\mu-1)(\lambda+\mu)}{\lambda \mu}} \nabla s \nabla \vartheta} \\
\leq H^{* *}(\lambda, \mu, x, y)\left(\int_{\vartheta_{0}}^{x}(x-\rho(s))\left(\omega_{1}(s)\right)^{\lambda} \nabla s\right)^{\frac{1}{\lambda}} \\
\quad \times\left(\int_{\vartheta_{0}}^{y}(y-\rho(\vartheta))\left(\omega_{2}(\vartheta)\right)^{\mu} \nabla \vartheta\right)^{\frac{1}{\mu}},
\end{gathered}
$$

where

$$
H^{* *}(\lambda, \mu, x, y)=\frac{1}{\lambda+\mu}\left(x-\vartheta_{0}\right)^{\frac{\lambda-1}{\lambda}}\left(y-\vartheta_{0}\right)^{\frac{\mu-1}{\mu}}
$$

For $\lambda=\mu=2$, this is Anderson's result [33, Remark 4].

In what follows, we give a further generalization of (32) obtained in Remark 3.5. Before giving our results, we presume that there are $\Phi$ and $\Psi$ which are real-valued, nonnegative, 
convex and submultiplicative functions defined on $[0, \infty)$. A function $\psi$ is submultiplicative if $\psi(s \vartheta) \leq \psi(s) \psi(\vartheta)$ for $s, \vartheta \geq 0$.

Theorem 3.6 Let $s, \vartheta, \vartheta_{0} \in \mathbb{T}$ and $\Pi(s), \Omega(\vartheta)$ be as in Theorem 3.1 and let $k(\xi), l(\xi)$ be two positive functions defined for $\xi \in\left[\vartheta_{0}, x\right]_{\mathbb{T}}$ and $\xi \in\left[\vartheta_{0}, y\right]_{\mathbb{T}}$. Suppose that

$$
K(s)=\int_{\vartheta_{0}}^{s} k(\xi) \nabla \xi \quad \text { and } \quad L(\vartheta)=\int_{\vartheta_{0}}^{\vartheta} l(\xi) \nabla \xi
$$

Then for $s \in\left[\vartheta_{0}, x\right]_{\mathbb{T}}$ and $\vartheta \in\left[\vartheta_{0}, y\right]_{\mathbb{T}}$, we have

$$
\begin{gathered}
\int_{\vartheta_{0}}^{x} \int_{\vartheta_{0}}^{y} \frac{\Phi(\Pi(s)) \Psi(\Omega(\vartheta))}{\mu\left(s-\vartheta_{0}\right)^{\frac{(\lambda-1)(\lambda+\mu)}{\lambda \mu}}+\lambda\left(\vartheta-\vartheta_{0}\right)^{\frac{(\mu-1)(\lambda+\mu)}{\lambda \mu}} \nabla s \nabla \vartheta} \\
\leq M(\lambda, \mu, x, y)\left(\int_{\vartheta_{0}}^{x}(x-\rho(s))\left(k(s) \Phi\left(\frac{\omega_{1}(s)}{k(s)}\right)\right)^{\lambda} \nabla s\right)^{\frac{1}{\lambda}} \\
\quad \times\left(\int_{\vartheta_{0}}^{y}(y-\rho(\vartheta))\left(l(\vartheta) \Psi\left(\frac{\omega_{2}(\vartheta)}{l(\vartheta)}\right)\right)^{\mu} \nabla \vartheta\right)^{\frac{1}{\mu}},
\end{gathered}
$$

where

$$
\begin{aligned}
M(\lambda, \mu, x, y)= & \frac{1}{\lambda+\mu}\left(\int_{\vartheta_{0}}^{x}\left(\frac{\Phi(K(s))}{K(s)}\right)^{\frac{\lambda}{\lambda-1}} \nabla s\right)^{\frac{\lambda-1}{\lambda}} \\
& \times\left(\int_{\vartheta_{0}}^{y}\left(\frac{\Psi(L(\vartheta))}{L(\vartheta)}\right)^{\frac{\mu}{\mu-1}} \nabla \vartheta\right)^{\frac{\mu-1}{\mu}} .
\end{aligned}
$$

Proof Using Jensen's inequality (12) and the properties of $\Phi$, we obtain

$$
\begin{aligned}
\Phi(\Pi(s)) & =\Phi\left(\frac{K(s) \int_{\vartheta_{0}}^{s} k(\xi) \frac{\omega_{1}(\xi)}{k(\xi)} \nabla \xi}{\int_{\vartheta_{0}}^{s} k(\xi) \nabla \xi}\right) \\
& \leq \Phi(K(s)) \Phi\left(\frac{\int_{\vartheta_{0}}^{s} k(\xi) \frac{\omega_{1}(\xi)}{k(\xi)} \nabla \xi}{\int_{\vartheta_{0}}^{s} k(\xi) \nabla \xi}\right) \\
& \leq \frac{\Phi(K(s))}{K(s)} \int_{\vartheta_{0}}^{s} k(\xi) \Phi\left(\frac{\omega_{1}(\xi)}{k(\xi)}\right) \nabla \xi .
\end{aligned}
$$

Further, by (11), we find that

$$
\Phi(\Pi(s)) \leq \frac{\Phi(K(s))}{K(s)}\left(s-\vartheta_{0}\right)^{\frac{\lambda-1}{\lambda}}\left(\int_{\vartheta_{0}}^{s}\left(k(\xi) \Phi\left(\frac{\omega_{1}(\xi)}{k(\xi)}\right)\right)^{\lambda} \nabla \xi\right)^{\frac{1}{\lambda}} .
$$

Analogously,

$$
\Psi(\Omega(\vartheta)) \leq \frac{\Psi(L(\vartheta))}{L(\vartheta)}\left(\vartheta-\vartheta_{0}\right)^{\frac{\mu-1}{\mu}}\left(\int_{\vartheta_{0}}^{\vartheta}\left(l(\xi) \Psi\left(\frac{\omega_{2}(\xi)}{l(\xi)}\right)\right)^{\mu} \nabla \xi\right)^{\frac{1}{\mu}} .
$$


By multiplying (36) and (37), we get

$$
\begin{aligned}
\Phi(\Pi(s)) \Psi(\Omega(\vartheta)) \\
\leq\left(s-\vartheta_{0}\right)^{\frac{\lambda-1}{\lambda}}\left(\vartheta-\vartheta_{0}\right)^{\frac{\mu-1}{\mu}}\left(\frac{\Phi(K(s))}{K(s)}\left(\int_{\vartheta_{0}}^{s}\left(k(\xi) \Phi\left(\frac{\omega_{1}(\xi)}{k(\xi)}\right)\right)^{\lambda} \nabla \xi\right)^{\frac{1}{\lambda}}\right) \\
\quad \times\left(\frac{\Psi(L(\vartheta))}{L(\vartheta)}\left(\int_{\vartheta}^{\vartheta}\left(l(\xi) \Psi\left(\frac{\omega_{2}(\xi)}{l(\xi)}\right)\right)^{\mu} \nabla \xi\right)^{\frac{1}{\mu}}\right) .
\end{aligned}
$$

Applying (24) on the term $\left(s-\vartheta_{0}\right)^{(\lambda-1) / \lambda} \times\left(\vartheta-\vartheta_{0}\right)^{(\mu-1) / \mu}$ gives

$$
\begin{aligned}
\Phi(\Pi(s)) \Psi(\Omega(\vartheta)) \\
\leq \frac{\lambda \mu}{\lambda+\mu}\left(\frac{\left(s-\vartheta_{0}\right)^{\frac{(\lambda-1)(\lambda+\mu)}{\lambda \mu}}}{\lambda}+\frac{\left(t-\vartheta_{0}\right)^{\frac{(\mu-1)(\lambda+\mu)}{\lambda \mu}}}{\mu}\right) \\
\quad \times\left(\frac{\Phi(K(s))}{K(s)}\left(\int_{\vartheta_{0}}^{s}\left(k(\xi) \Phi\left(\frac{\omega_{1}(\xi)}{k(\xi)}\right)\right)^{\lambda} \nabla \xi\right)^{\frac{1}{\lambda}}\right) \\
\quad \times\left(\frac{\Psi(L(\vartheta))}{L(\vartheta)}\left(\int_{\vartheta_{0}}^{\vartheta}\left(l(\xi) \Psi\left(\frac{\omega_{2}(\xi)}{l(\xi)}\right)\right)^{\mu} \nabla \xi\right)^{\frac{1}{\mu}}\right) .
\end{aligned}
$$

From (39), we observe that

$$
\begin{gathered}
\frac{\Phi(\Pi(s)) \Psi(\Omega(\vartheta))}{\mu\left(s-\vartheta_{0}\right) \frac{(\lambda-1)(\lambda+\mu)}{\lambda \mu}+\lambda\left(\vartheta-\vartheta_{0}\right)^{\frac{(\mu-1)(\lambda+\mu)}{\lambda \mu}}} \\
\leq \frac{1}{\lambda+\mu}\left(\frac{\Phi(K(s))}{K(s)}\left(\int_{\vartheta_{0}}^{s}\left(k(\xi) \Phi\left(\frac{\omega_{1}(\xi)}{k(\xi)}\right)\right)^{\lambda} \nabla \xi\right)^{\frac{1}{\lambda}}\right) \\
\times\left(\frac{\Psi(L(\vartheta))}{L(\vartheta)}\left(\int_{\vartheta_{0}}^{\vartheta}\left(l(\xi) \Psi\left(\frac{\omega_{2}(\xi)}{l(\xi)}\right)\right)^{\mu} \nabla \xi\right)^{\frac{1}{\mu}}\right) .
\end{gathered}
$$

Integrating both sides of (40) and using (11) again with indices $\lambda, \lambda /(\lambda-1)$ and $\mu, \mu /(\mu-1)$, we find that

$$
\begin{aligned}
\int_{\vartheta_{0}}^{x} \int_{\vartheta_{0}}^{y} \frac{\Phi(\Pi(s)) \Psi(\Omega(\vartheta))}{\mu\left(s-\vartheta_{0}\right)^{\frac{(\lambda-1)(\lambda+\mu)}{\lambda \mu}}+\lambda\left(\vartheta-\vartheta_{0}\right)^{\frac{(\mu-1)(\lambda+\mu)}{\lambda \mu}} \nabla s \nabla \vartheta} \\
\leq \frac{1}{\lambda+\mu}\left(\int_{\vartheta_{0}}^{x}\left(\frac{\Phi(K(s))}{K(s)}\right)^{\frac{\lambda}{\lambda-1}} \nabla s\right)^{\frac{\lambda-1}{\lambda}}\left(\int_{\vartheta_{0}}^{x} \int_{\vartheta_{0}}^{s}\left(k(\xi) \Phi\left(\frac{\omega_{1}(\xi)}{k(\xi)}\right)\right)^{\lambda} \nabla \xi \nabla s\right)^{\frac{1}{\lambda}} \\
\quad \times\left(\int_{\vartheta_{0}}^{y}\left(\frac{\Psi(L(\vartheta))}{L(\vartheta)}\right)^{\frac{\mu}{\mu-1}} \nabla \vartheta\right)^{\frac{\mu-1}{\mu}}\left(\int_{\vartheta_{0}}^{y} \int_{\vartheta_{0}}^{\vartheta}\left(l(\xi) \Psi\left(\frac{\omega_{2}(\xi)}{l(\xi)}\right)\right)^{\mu} \nabla \xi \nabla \vartheta\right)^{\frac{1}{\mu}} .
\end{aligned}
$$

Applying Lemma 2.5 to (41), we get

$$
\begin{aligned}
& \int_{\vartheta_{0}}^{x} \int_{\vartheta_{0}}^{y} \frac{\Phi(\Pi(s)) \Psi(\Omega(\vartheta))}{\mu\left(s-\vartheta_{0}\right)^{\frac{(\lambda-1)(\lambda+\mu)}{\lambda \mu}}+\lambda\left(\vartheta-\vartheta_{0}\right)^{\frac{(\mu-1)(\lambda+\mu)}{\lambda \mu}} \nabla s \nabla \vartheta} \\
& \quad \leq \frac{1}{\lambda+\mu}\left(\int_{\vartheta_{0}}^{x}\left(\frac{\Phi(K(s))}{K(s)}\right)^{\frac{\lambda}{\lambda-1}} \nabla s\right)^{\frac{\lambda-1}{\lambda}}\left(\int_{\vartheta_{0}}^{y}\left(\frac{\Psi(L(\vartheta))}{L(\vartheta)}\right)^{\frac{\mu}{\mu-1}} \nabla \vartheta\right)^{\frac{\mu-1}{\mu}}
\end{aligned}
$$




$$
\begin{aligned}
& \times\left(\int_{\vartheta_{0}}^{x}(x-\rho(s))\left(k(s) \Phi\left(\frac{\omega_{1}(s)}{k(s)}\right)\right)^{\lambda} \nabla s\right)^{\frac{1}{\lambda}} \\
& \times\left(\int_{\vartheta_{0}}^{y}(y-\rho(\vartheta))\left(l(\vartheta) \Psi\left(\frac{\omega_{2}(\vartheta)}{l(\vartheta)}\right)\right)^{\mu} \nabla \vartheta\right)^{\frac{1}{\mu}} \\
= & M(\lambda, \mu, x, y)\left(\int_{\vartheta_{0}}^{x}(x-\rho(s))\left(k(s) \Phi\left(\frac{\omega_{1}(s)}{k(s)}\right)\right)^{\lambda} \nabla s\right)^{\frac{1}{\lambda}} \\
& \times\left(\int_{\vartheta_{0}}^{y}(y-\rho(\vartheta))\left(l(\vartheta) \Psi\left(\frac{\omega_{2}(\vartheta)}{l(\vartheta)}\right)\right)^{\mu} \nabla \vartheta\right)^{\frac{1}{\mu}},
\end{aligned}
$$

which is (34).

Corollary 3.2 If we take $1 / \lambda+1 / \mu=1$ in (34), then we get

$$
\begin{aligned}
& \int_{\vartheta_{0}}^{x} \int_{\vartheta_{0}}^{y} \frac{\Phi(\Pi(s)) \Psi(\Omega(\vartheta))}{\mu\left(s-\vartheta_{0}\right)^{\lambda-1}+\lambda\left(\vartheta-\vartheta_{0}\right)^{\mu-1}} \nabla s \nabla \vartheta \\
& \leq M^{*}(\lambda, \mu, x, y)\left(\int_{\vartheta_{0}}^{x}(x-\rho(s))\left(k(s) \Phi\left(\frac{\omega_{1}(s)}{k(s)}\right)\right)^{\lambda} \nabla s\right)^{\frac{1}{\lambda}} \\
& \quad \times\left(\int_{\vartheta_{0}}^{y}(y-\rho(\vartheta))\left(l(\vartheta) \Psi\left(\frac{\omega_{2}(\vartheta)}{l(\vartheta)}\right)\right)^{\mu} \nabla \vartheta\right)^{\frac{1}{\mu}},
\end{aligned}
$$

where

$$
\begin{aligned}
M^{*}(\lambda, \mu, x, y)= & \frac{1}{\lambda \mu}\left(\int_{\vartheta_{0}}^{x}\left(\frac{\Phi(K(s))}{K(s)}\right)^{\frac{\lambda}{\lambda-1}} \nabla s\right)^{\frac{\lambda-1}{\lambda}} \\
& \times\left(\int_{\vartheta_{0}}^{y}\left(\frac{\Psi(L(\vartheta))}{L(\vartheta)}\right)^{\frac{\mu}{\mu-1}} \nabla \vartheta\right)^{\frac{\mu-1}{\mu}} .
\end{aligned}
$$

Remark 3.7 As a particular case of Corollary 3.2, if $\lambda=\mu=2$, then we get

$$
\begin{aligned}
& \int_{\vartheta_{0}}^{x} \int_{\vartheta_{0}}^{y} \frac{\Phi(\Pi(s)) \Psi(\Omega(\vartheta))}{s+\vartheta-2 \vartheta_{0}} \nabla s \nabla \vartheta \\
& \leq M^{* *}(x, y)\left(\int_{\vartheta_{0}}^{x}(x-\rho(s))\left(k(s) \Phi\left(\frac{\omega_{1}(s)}{k(s)}\right)\right)^{2} \nabla s\right)^{\frac{1}{2}} \\
& \quad \times\left(\int_{\vartheta_{0}}^{y}(y-\rho(\vartheta))\left(l(\vartheta) \Psi\left(\frac{\omega_{2}(\vartheta)}{l(\vartheta)}\right)\right)^{2} \nabla \vartheta\right)^{\frac{1}{2}}
\end{aligned}
$$

where

$$
M^{* *}(x, y)=\frac{1}{2}\left(\int_{\vartheta_{0}}^{x}\left(\frac{\Phi(K(s))}{K(s)}\right)^{2} \nabla s\right)^{\frac{1}{2}}\left(\int_{\vartheta_{0}}^{y}\left(\frac{\Psi(L(\vartheta))}{L(\vartheta)}\right)^{2} \nabla \vartheta\right)^{\frac{1}{2}},
$$

which is [33, Theorem 3.5]. 
Remark 3.8 As a particular case of Theorem 3.6 if $\mathbb{T}=\mathbb{Z}, \vartheta_{0}=0$, then $\rho(u)=u-1$ and (34) reduces to

$$
\begin{aligned}
\sum_{s=1}^{p} & \sum_{\vartheta=1}^{q} \frac{\Phi\left(\Pi_{s}\right) \Psi\left(\Omega_{\vartheta}\right)}{\mu s^{\frac{(\lambda-1)(\lambda+\mu)}{\lambda \mu}}+\lambda \vartheta \frac{(\mu-1)(\lambda+\mu)}{\lambda \mu}} \\
\leq & M_{0}(\lambda, \mu, p, q)\left(\sum_{s=1}^{p}(p-s+1)\left(k_{s} \Phi\left(\frac{\omega_{s}}{k_{s}}\right)\right)^{\lambda}\right)^{\frac{1}{\lambda}} \\
& \times\left(\sum_{\vartheta=1}^{q}(q-\vartheta+1)\left(l_{\vartheta} \Phi\left(\frac{\omega_{\vartheta}}{l_{\vartheta}}\right)\right)^{\mu}\right)^{\frac{1}{\mu}}
\end{aligned}
$$

where

$$
M_{0}(\lambda, \mu, p, q)=\frac{1}{\lambda+\mu}\left(\sum_{s=1}^{p}\left(\frac{\Phi\left(K_{s}\right)}{K_{s}}\right)^{\frac{\lambda}{\lambda-1}}\right)^{\frac{\lambda-1}{\lambda}}\left(\sum_{\vartheta=1}^{q}\left(\frac{\Psi\left(L_{\vartheta}\right)}{L_{\vartheta}}\right)^{\frac{\mu}{\mu-1}}\right)^{\frac{\mu-1}{\mu}}
$$

which is [13, Theorem 2.2].

Remark 3.9 As a particular case of Theorem 3.6 if $\mathbb{T}=\mathbb{R}, t_{0}=0$, then $\rho(u)=u$ and (34) reduces to

$$
\begin{aligned}
\int_{0}^{x} \int_{0}^{y} \frac{\Phi(\Pi(s)) \Psi(\Omega(\vartheta))}{\mu s^{\frac{(\lambda-1)(\lambda+\mu)}{\lambda \mu}}+\lambda \vartheta^{\frac{(\mu-1)(\lambda+\mu)}{\lambda \mu}}} d s d t \\
\leq M_{0}^{*}(\lambda, \mu, x, y)\left(\int_{0}^{x}(x-s)\left(k(s) \Phi\left(\frac{\omega_{1}(s)}{k(s)}\right)\right)^{\lambda} d s\right)^{\frac{1}{\lambda}} \\
\quad \times\left(\int_{0}^{y}(y-\vartheta)\left(l(\vartheta) \Psi\left(\frac{\omega_{2}(\vartheta)}{l(\vartheta)}\right)\right)^{\mu} d \vartheta\right)^{\frac{1}{\mu}}
\end{aligned}
$$

where

$$
\begin{aligned}
M_{0}^{*}(\lambda, \mu, x, y)= & \frac{1}{\lambda+\mu}\left(\int_{0}^{x}\left(\frac{\Phi(K(s))}{K(s)}\right)^{\frac{\lambda}{\lambda-1}} d s\right)^{\frac{\lambda-1}{\lambda}} \\
& \times\left(\int_{0}^{y}\left(\frac{\Psi(L(\vartheta))}{L(\vartheta)}\right)^{\frac{\mu}{\mu-1}} d \vartheta\right)^{\frac{\mu-1}{\mu}},
\end{aligned}
$$

which is [13, Theorem 3.2].

Our next outcome deals with a further generalization of the inequality in (34).

Theorem 3.10 Let $s, \vartheta, \vartheta_{0} \in \mathbb{T}$, and $\omega_{1}, \omega_{2}$ be as in Theorem 3.1. Define

$$
\Pi(s)=\frac{1}{s-\vartheta_{0}} \int_{\vartheta_{0}}^{s} \omega_{1}(\xi) \nabla \xi \quad \text { and } \quad \Omega(\vartheta)=\frac{1}{\vartheta-\vartheta_{0}} \int_{\vartheta_{0}}^{\vartheta} \omega_{2}(\xi) \nabla \xi
$$


Then for $s \in\left[\vartheta_{0}, x\right]_{\mathbb{T}}$ and $\vartheta \in\left[\vartheta_{0}, y\right]_{\mathbb{T}}$, we have

$$
\begin{gathered}
\int_{\vartheta_{0}}^{x} \int_{\vartheta_{0}}^{y} \frac{\Phi(\Pi(s)) \Psi(\Omega(\vartheta))\left(s-\vartheta_{0}\right)\left(\vartheta-\vartheta_{0}\right)}{\mu\left(s-\vartheta_{0}\right) \frac{(\lambda-1)(\lambda+\mu)}{\lambda \mu}+\lambda\left(\vartheta-\vartheta_{0}\right) \frac{(\mu-1)(\lambda+\mu)}{\lambda \mu}} \nabla s \nabla \vartheta \\
\leq N(\lambda, \mu, x, y)\left(\int_{\vartheta_{0}}^{x}(x-\rho(s))\left(\Phi\left(\omega_{1}(s)\right)\right)^{\lambda} \nabla s\right)^{\frac{1}{\lambda}} \\
\quad \times\left(\int_{\vartheta_{0}}^{y}(y-\rho(\vartheta))\left(\Psi\left(\omega_{2}(\vartheta)\right)\right)^{\mu} \nabla \vartheta\right)^{\frac{1}{\mu}},
\end{gathered}
$$

where

$$
N(\lambda, \mu, x, y)=\frac{1}{\lambda+\mu}\left(x-\vartheta_{0}\right)^{\frac{\lambda-1}{\lambda}}\left(y-\vartheta_{0}\right)^{\frac{\mu-1}{\mu}} .
$$

Proof Based on the assumptions and the inequality of Jensen (12), we can see that

$$
\begin{aligned}
\Phi(\Pi(s)) & =\Phi\left(\frac{1}{s-\vartheta_{0}} \int_{\vartheta_{0}}^{s} \omega_{1}(\xi) \nabla \xi\right) \\
& \leq \frac{1}{s-\vartheta_{0}} \int_{\vartheta_{0}}^{s} \Phi\left(\omega_{1}(\xi)\right) \nabla \xi .
\end{aligned}
$$

By applying (11) to (48) with indices $\lambda, \lambda /(\lambda-1)$, we have

$$
\Phi(\Pi(s)) \leq \frac{1}{s-\vartheta_{0}}\left(s-\vartheta_{0}\right)^{\frac{\lambda-1}{\lambda}}\left(\int_{\vartheta_{0}}^{s}\left(\Phi\left(\omega_{1}(\xi)\right)\right)^{\lambda} \nabla \xi\right)^{\frac{1}{\lambda}} .
$$

This implies that

$$
\Phi(\Pi(s))\left(s-\vartheta_{0}\right) \leq\left(s-\vartheta_{0}\right)^{\frac{\lambda-1}{\lambda}}\left(\int_{\vartheta_{0}}^{s}\left(\Phi\left(\omega_{1}(\xi)\right)\right)^{\lambda} \nabla \xi\right)^{\frac{1}{\lambda}} .
$$

Analogously,

$$
\Psi(\Omega(\vartheta))\left(\vartheta-\vartheta_{0}\right) \leq\left(\vartheta-\vartheta_{0}\right)^{\frac{\mu-1}{\mu}}\left(\int_{\vartheta_{0}}^{t}\left(\Psi\left(\omega_{2}(\xi)\right)\right)^{\mu} \nabla \xi\right)^{\frac{1}{\mu}} .
$$

From (50) and (51), we get

$$
\begin{aligned}
\Phi(\Pi(s)) \Psi(\Omega(\vartheta))\left(s-\vartheta_{0}\right)\left(\vartheta-\vartheta_{0}\right) \\
\leq\left(s-\vartheta_{0}\right)^{\frac{\lambda-1}{\lambda}}\left(\vartheta-\vartheta_{0}\right)^{\frac{\mu-1}{\mu}}\left(\int_{\vartheta_{0}}^{s}\left(\Phi\left(\omega_{1}(\xi)\right)\right)^{\lambda} \nabla \xi\right)^{\frac{1}{\lambda}} \\
\quad \times\left(\int_{\vartheta_{0}}^{t}\left(\Psi\left(\omega_{2}(\xi)\right)\right)^{\mu} \nabla \xi\right)^{\frac{1}{\mu}} .
\end{aligned}
$$


Applying (24) to the term $\left(s-\vartheta_{0}\right)^{(\lambda-1) / \lambda} \times\left(\vartheta-\vartheta_{0}\right)^{(\mu-1) / \mu}$ gives

$$
\begin{aligned}
& \Phi(\Pi(s)) \Psi(\Omega(\vartheta))\left(s-\vartheta_{0}\right)\left(\vartheta-\vartheta_{0}\right) \\
& \leq \frac{\lambda \mu}{\lambda+\mu}\left(\frac{\left(s-\vartheta_{0}\right)^{\frac{(\lambda-1)(\lambda+\mu)}{\lambda \mu}}}{\lambda}+\frac{\left(\vartheta-\vartheta_{0}\right)^{\frac{(\mu-1)(\lambda+\mu)}{\lambda \mu}}}{\mu}\right) \\
& \quad \times\left(\int_{\vartheta_{0}}^{s}\left(\Phi\left(\omega_{1}(\xi)\right)\right)^{\lambda} \nabla \xi\right)^{\frac{1}{\lambda}}\left(\int_{\vartheta_{0}}^{t}\left(\Psi\left(\omega_{2}(\xi)\right)\right)^{\mu} \nabla \xi\right)^{\frac{1}{\mu}} .
\end{aligned}
$$

From (53), we have

$$
\begin{aligned}
& \frac{\Phi(\Pi(s)) \Psi(\Omega(\vartheta))\left(s-\vartheta_{0}\right)\left(\vartheta-\vartheta_{0}\right)}{\mu\left(s-\vartheta_{0}\right)^{\frac{(\lambda-1)(\lambda+\mu)}{\lambda \mu}}+\lambda\left(\vartheta-\vartheta_{0}\right)^{\frac{(\mu-1)(\lambda+\mu)}{\lambda \mu}}} \\
& \leq \frac{1}{\lambda+\mu}\left(\int_{\vartheta_{0}}^{s}\left(\Phi\left(\omega_{1}(\xi)\right)\right)^{\lambda} \nabla \xi\right)^{\frac{1}{\lambda}}\left(\int_{\vartheta_{0}}^{t}\left(\Psi\left(\omega_{2}(\xi)\right)\right)^{\mu} \nabla \xi\right)^{\frac{1}{\mu}} .
\end{aligned}
$$

Integrating both sides of (54) and using (11) again with indices $\lambda, \lambda /(\lambda-1)$ and $\mu, \mu /(\mu-1)$, we get

$$
\begin{aligned}
\int_{\vartheta_{0}}^{x} \int_{\vartheta_{0}}^{y} \frac{\Phi(\Pi(s)) \Psi(\Omega(\vartheta))\left(s-\vartheta_{0}\right)\left(\vartheta-\vartheta_{0}\right)}{\mu\left(s-\vartheta_{0}\right)^{\frac{(\lambda-1)(\lambda+\mu)}{\lambda \mu}}+\lambda\left(\vartheta-\vartheta_{0}\right)^{\frac{(\mu-1)(\lambda+\mu)}{\lambda \mu}} \nabla s \nabla \vartheta} \\
\leq \frac{1}{\lambda+\mu}\left(x-\vartheta_{0}\right)^{\frac{\lambda-1}{\lambda}}\left(y-\vartheta_{0}\right)^{\frac{\mu-1}{\mu}}\left(\int_{\vartheta_{0}}^{x}\left(\int_{\vartheta_{0}}^{s}\left(\Phi\left(\omega_{1}(\xi)\right)\right)^{\lambda} \nabla \xi\right) \nabla s\right)^{\frac{1}{\lambda}} \\
\quad \times\left(\int_{\vartheta_{0}}^{y}\left(\int_{\vartheta_{0}}^{\vartheta}\left(\Psi\left(\omega_{2}(\xi)\right)\right)^{\mu} \nabla \xi\right) \nabla \vartheta\right)^{\frac{1}{\mu}} .
\end{aligned}
$$

Applying Lemma 2.5 to (55), we find that

$$
\begin{aligned}
& \int_{\vartheta_{0}}^{x} \int_{\vartheta_{0}}^{y} \frac{\Phi(\Pi(s)) \Psi(\Omega(\vartheta))\left(s-\vartheta_{0}\right)\left(\vartheta-\vartheta_{0}\right)}{\mu\left(s-\vartheta_{0}\right)^{\frac{(\lambda-1)(\lambda+\mu)}{\lambda \mu}}+\lambda\left(\vartheta-\vartheta_{0}\right)^{\frac{(\mu-1)(\lambda+\mu)}{\lambda \mu}}} \nabla s \nabla \vartheta \\
& \leq \frac{1}{\lambda+\mu}\left(x-\vartheta_{0}\right)^{\frac{\lambda-1}{\lambda}}\left(y-\vartheta_{0}\right)^{\frac{\mu-1}{\mu}} \\
& \times\left(\int_{\vartheta_{0}}^{x}(x-\rho(s))\left(\Phi\left(\omega_{1}(s)\right)\right)^{\lambda} \nabla s\right)^{\frac{1}{\lambda}} \\
& \times\left(\int_{\vartheta_{0}}^{y}(y-\rho(\vartheta))\left(\Psi\left(\omega_{2}(\vartheta)\right)\right)^{\mu} \nabla \vartheta\right)^{\frac{1}{\mu}} \\
&= N(\lambda, \mu, x, y)\left(\int_{\vartheta_{0}}^{x}(x-\rho(s))\left(\Phi\left(\omega_{1}(s)\right)\right)^{\lambda} \nabla s\right)^{\frac{1}{\lambda}} \\
& \times\left(\int_{\vartheta_{0}}^{y}(y-\rho(\vartheta))\left(\Psi\left(\omega_{2}(\vartheta)\right)\right)^{\mu} \nabla \vartheta\right)^{\frac{1}{\mu}},
\end{aligned}
$$

which is (47). 
Corollary 3.3 If we take $1 / \lambda+1 / \mu=1$ in (47), then we get

$$
\begin{gathered}
\int_{\vartheta_{0}}^{x} \int_{\vartheta_{0}}^{y} \frac{\Phi(\Pi(s)) \Psi(\Omega(\vartheta))\left(s-\vartheta_{0}\right)\left(\vartheta-\vartheta_{0}\right)}{\mu\left(s-\vartheta_{0}\right)^{\lambda-1}+\lambda\left(\vartheta-\vartheta_{0}\right)^{\mu-1}} \nabla s \nabla \vartheta \\
\leq N^{*}(\lambda, \mu, x, y)\left(\int_{\vartheta_{0}}^{x}(x-\rho(s))\left(\Phi\left(\omega_{1}(s)\right)\right)^{\lambda} \nabla s\right)^{\frac{1}{\lambda}} \\
\quad \times\left(\int_{\vartheta_{0}}^{y}(y-\rho(\vartheta))\left(\Psi\left(\omega_{2}(\vartheta)\right)\right)^{\mu} \nabla \vartheta\right)^{\frac{1}{\mu}},
\end{gathered}
$$

where

$$
N^{*}(\lambda, \mu, x, y)=\frac{1}{\lambda \mu}\left(x-\vartheta_{0}\right)^{\frac{\lambda-1}{\lambda}}\left(y-\vartheta_{0}\right)^{\frac{\mu-1}{\mu}} .
$$

Remark 3.11 As a particular case of Corollary 3.3, if $\lambda=\mu=2$, then we get

$$
\begin{aligned}
\int_{\vartheta_{0}}^{x} \int_{\vartheta_{0}}^{y} \frac{\Phi(\Pi(s)) \Psi(\Omega(\vartheta))\left(s-\vartheta_{0}\right)\left(\vartheta-\vartheta_{0}\right)}{s+\vartheta-2 \vartheta_{0}} \nabla s \nabla \vartheta \\
\leq \frac{1}{2}\left(\left(x-\vartheta_{0}\right) \int_{\vartheta_{0}}^{x}(x-\rho(s))\left(\Phi\left(\omega_{1}(s)\right)\right)^{2} \nabla s\right)^{\frac{1}{2}} \\
\quad \times\left(\left(y-\vartheta_{0}\right) \int_{\vartheta_{0}}^{y}(y-\rho(\vartheta))\left(\Psi\left(\omega_{2}(\vartheta)\right)\right)^{2} \nabla \vartheta\right)^{\frac{1}{2}},
\end{aligned}
$$

which is [33, Theorem 3.6].

Remark 3.12 As a particular case of Theorem 3.10, if $\mathbb{T}=\mathbb{Z}, \vartheta_{0}=0$, then $\rho(u)=u-1$ and (47) reduces to

$$
\begin{aligned}
& \sum_{s=1}^{p} \sum_{\vartheta=1}^{q} \frac{\Phi\left(\Pi_{s}\right) \Psi\left(\Omega_{\vartheta}\right) s \vartheta}{\mu s^{\frac{(\lambda-1)(\lambda+\mu)}{\lambda \mu}}+\lambda \vartheta \frac{(\mu-1)(\lambda+\mu)}{\lambda \mu}} \\
& \quad \leq N_{0}(\lambda, \mu, p, q)\left(\sum_{s=1}^{p}(p-s+1)\left(\Phi\left(\omega_{s}\right)\right)^{\lambda}\right)^{\frac{1}{\lambda}}\left(\sum_{\vartheta=1}^{q}(q-\vartheta+1)\left(\Phi\left(\omega_{\vartheta}\right)\right)^{\mu}\right)^{\frac{1}{\mu}},
\end{aligned}
$$

where

$$
N_{0}(\lambda, \mu, p, q)=\frac{1}{\lambda+\mu} p^{\frac{\lambda-1}{\lambda}} q^{\frac{\mu-1}{\mu}},
$$

which is [13, Theorem 2.3].

Remark 3.13 As a particular state of Theorem 3.10, if $\mathbb{T}=\mathbb{R}, t_{0}=0$, then $\rho(u)=u$ and (47) reduces to

$$
\begin{aligned}
& \int_{0}^{x} \int_{0}^{y} \frac{\Phi(\Pi(s)) \Psi(\Omega(\vartheta)) s \vartheta}{\mu s^{\frac{(\lambda-1)(\lambda+\mu)}{\lambda \mu}}+\lambda \vartheta^{\frac{(\mu-1)(\lambda+\mu)}{\lambda \mu}} d s d \vartheta} \\
& \leq N_{0}^{*}(\lambda, \mu, x, y)\left(\int_{0}^{x}(x-s)\left(\Phi\left(\omega_{1}(s)\right)\right)^{\lambda} d s\right)^{\frac{1}{\lambda}} \\
& \quad \times\left(\int_{0}^{y}(y-\vartheta)\left(\Psi\left(\omega_{2}(\vartheta)\right)\right)^{\mu} d \vartheta\right)^{\frac{1}{\mu}},
\end{aligned}
$$


where

$$
N_{0}^{*}(\lambda, \mu, x, y)=\frac{1}{\lambda+\mu} x^{\frac{\lambda-1}{\lambda}} y^{\frac{\mu-1}{\mu}},
$$

which is [13, Theorem 3.3].

Theorem 3.14 Let $s, \vartheta, \vartheta_{0} \in \mathbb{T}$ and $\omega_{1}, \omega_{2}, k, l, H, L$ be as in Theorem 3.6. Define

$$
\Pi(s)=\frac{1}{K(s)} \int_{\vartheta_{0}}^{s} k(\xi) \omega_{1}(\xi) \nabla \xi \quad \text { and } \quad \Omega(\vartheta)=\frac{1}{L(\vartheta)} \int_{\vartheta_{0}}^{\vartheta} l(\xi) \omega_{2}(\xi) \nabla \xi .
$$

Then for $s \in\left[\vartheta_{0}, y\right]_{\mathbb{T}}$ and $\vartheta \in\left[\vartheta_{0}, x\right]_{\mathbb{T}}$, we get

$$
\begin{gathered}
\int_{\vartheta_{0}}^{x} \int_{\vartheta_{0}}^{y} \frac{K(s) L(\vartheta) \Phi(\Pi(s)) \Psi(\Omega(\vartheta))}{\mu\left(s-\vartheta_{0}\right)^{\frac{(\lambda-1)(\lambda+\mu)}{\lambda \mu}}+\lambda(\vartheta-\vartheta)^{\frac{((-1)(\lambda+\mu)}{\lambda \mu}}} \nabla s \nabla \vartheta \\
\leq W(\lambda, \mu, x, y)\left(\int_{\vartheta_{0}}^{x}(x-\rho(s))\left(k(s) \Phi\left(\omega_{1}(s)\right)\right)^{\lambda} \nabla s\right)^{\frac{1}{\lambda}} \\
\quad \times\left(\int_{\vartheta_{0}}^{y}(y-\rho(\vartheta))\left(l(\vartheta) \Psi\left(\omega_{2}(\vartheta)\right)\right)^{\mu} \nabla \vartheta\right)^{\frac{1}{\mu}},
\end{gathered}
$$

where

$$
W(\lambda, \mu, x, y)=\frac{1}{\lambda+\mu}\left(x-\vartheta_{0}\right)^{\frac{\lambda-1}{\lambda}}\left(y-\vartheta_{0}\right)^{\frac{\mu-1}{\mu}} .
$$

Proof Based on the assumptions and the inequality of Jensen (12), we find that

$$
\begin{aligned}
\Phi(\Pi(s)) & =\Phi\left(\frac{1}{K(s)} \int_{\vartheta_{0}}^{s} k(\xi) \omega_{1}(\xi) \nabla \xi\right) \\
& \leq \frac{1}{K(s)} \int_{\vartheta_{0}}^{s} k(\xi) \Phi\left(\omega_{1}(\xi)\right) \nabla \xi .
\end{aligned}
$$

By applying (11) to (62) with indices $\lambda, \lambda /(\lambda-1)$, we have

$$
\Phi(\Pi(s)) \leq \frac{1}{K(s)}\left(s-\vartheta_{0}\right)^{\frac{\lambda-1}{\lambda}}\left(\int_{\vartheta_{0}}^{s}\left(k(\xi) \Phi\left(\omega_{1}(\xi)\right)\right)^{\lambda} \nabla \xi\right)^{\frac{1}{\lambda}} .
$$

From (63), we get

$$
\Phi(\Pi(s)) K(s) \leq\left(s-\vartheta_{0}\right)^{\frac{\lambda-1}{\lambda}}\left(\int_{\vartheta_{0}}^{s}\left(k(\xi) \Phi\left(\omega_{1}(\xi)\right)\right)^{\lambda} \nabla \xi\right)^{\frac{1}{\lambda}} .
$$

Similarly, we also obtain

$$
\Psi\left(\Omega(\vartheta) L(\vartheta) \leq\left(\vartheta-\vartheta_{0}\right)^{\frac{\mu-1}{\mu}}\left(\int_{\vartheta_{0}}^{\vartheta}\left(l(\xi) \Psi\left(\omega_{2}(\xi)\right)\right)^{\mu} \nabla \xi\right)^{\frac{1}{\mu}} .\right.
$$


From (64) and (65), we find that

$$
\begin{aligned}
& K(s) L(\vartheta) \Phi(\Pi(s)) \Psi(\Omega(\vartheta) \\
& \leq\left(s-\vartheta_{0}\right)^{\frac{\lambda-1}{\lambda}}\left(\vartheta-\vartheta_{0}\right)^{\frac{\mu-1}{\mu}}\left(\int_{\vartheta_{0}}^{s}\left(k(\xi) \Phi\left(\omega_{1}(\xi)\right)\right)^{\lambda} \nabla \xi\right)^{\frac{1}{\lambda}} \\
& \quad \times\left(\int_{\vartheta_{0}}^{\vartheta}\left(l(\xi) \Psi\left(\omega_{2}(\xi)\right)\right)^{\mu} \nabla \xi\right)^{\frac{1}{\mu}} .
\end{aligned}
$$

Applying (24) to the term $\left(s-\vartheta_{0}\right)^{(\lambda-1) / \lambda} \times\left(\vartheta-\vartheta_{0}\right)^{(\mu-1) / \mu}$ gives

$$
\begin{aligned}
& K(s) L(\vartheta) \Phi(\Pi(s)) \Psi(\Omega(\vartheta) \\
& \leq \frac{\lambda \mu}{\lambda+\mu}\left(\frac{\left(s-\vartheta_{0}\right)^{\frac{(\lambda-1)(\lambda+\mu)}{\lambda \mu}}}{\lambda}+\frac{\left(\vartheta-\vartheta_{0}\right)^{\frac{(\mu-1)(\lambda+\mu)}{\lambda \mu}}}{\mu}\right) \\
& \quad \times\left(\int_{\vartheta_{0}}^{s}\left(k(\xi) \Phi\left(\omega_{1}(\xi)\right)\right)^{\lambda} \nabla \xi\right)^{\frac{1}{\lambda}}\left(\int_{\vartheta_{0}}^{\vartheta}\left(l(\xi) \Psi\left(\omega_{2}(\xi)\right)\right)^{\mu} \nabla \xi\right)^{\frac{1}{\mu}} .
\end{aligned}
$$

This implies that

$$
\begin{aligned}
& \frac{K(s) L(\vartheta) \Phi(\Pi(s)) \Psi(\Omega(\vartheta)}{\mu\left(s-\vartheta_{0}\right)^{\frac{(\lambda-1)(\lambda+\mu)}{\lambda \mu}}+\lambda\left(\vartheta-\vartheta_{0}\right)^{\frac{(\mu-1)(\lambda+\mu)}{\lambda \mu}}} \\
& \leq \frac{1}{\lambda+\mu}\left(\int_{\vartheta_{0}}^{s}\left(k(\xi) \Phi\left(\omega_{1}(\xi)\right)\right)^{\lambda} \nabla \xi\right)^{\frac{1}{\lambda}}\left(\int_{\vartheta_{0}}^{\vartheta}\left(l(\xi) \Psi\left(\omega_{2}(\xi)\right)\right)^{\mu} \nabla \xi\right)^{\frac{1}{\mu}} .
\end{aligned}
$$

Integrating both sides of (68) and using (11) again with indices $\lambda, \lambda /(\lambda-1)$ and $\mu, \mu /(\mu-1)$, we get

$$
\begin{aligned}
& \int_{\vartheta_{0}}^{x} \int_{\vartheta_{0}}^{y} \frac{K(s) L(\vartheta) \Phi(\Pi(s)) \Psi(\Omega(\vartheta)}{\mu\left(s-\vartheta_{0}\right)^{\frac{(\lambda-1)(\lambda+\mu)}{\lambda \mu}}+\lambda\left(\vartheta-\vartheta_{0}\right)^{\frac{(\mu-1)(\lambda+\mu)}{\lambda \mu}}} \nabla s \nabla \vartheta \\
& \leq \frac{1}{\lambda+\mu}\left(s-\vartheta_{0}\right)^{\frac{\lambda-1}{\lambda}}\left(\vartheta-\vartheta_{0}\right)^{\frac{\mu-1}{\mu}}\left(\int_{\vartheta_{0}}^{x}\left(\int_{\vartheta_{0}}^{s}\left(k(\xi) \Phi\left(\omega_{1}(\xi)\right)\right)^{\lambda} \nabla \xi\right) \nabla s\right)^{\frac{1}{\lambda}} \\
& \quad \times\left(\int_{\vartheta_{0}}^{y}\left(\int_{\vartheta_{0}}^{\vartheta}\left(l(\xi) \Psi\left(\omega_{2}(\xi)\right)\right)^{\mu} \nabla \xi\right) \nabla \vartheta\right)^{\frac{1}{\mu}} .
\end{aligned}
$$

Applying Lemma 2.5 to (69), we find that

$$
\begin{gathered}
\int_{\vartheta_{0}}^{x} \int_{\vartheta_{0}}^{y} \frac{K(s) L(\vartheta) \Phi(\Pi(s)) \Psi(\Omega(\vartheta)}{\mu\left(s-\vartheta_{0}\right)^{\frac{(\lambda-1)(\lambda+\mu)}{\lambda \mu}}+\lambda\left(\vartheta-\vartheta_{0}\right)^{\frac{(\mu-1)(\lambda+\mu)}{\lambda \mu}}} \nabla s \nabla \vartheta \\
\leq W(\lambda, \mu, x, y)\left(\int_{\vartheta_{0}}^{x}(x-\rho(s))\left(k(s) \Phi\left(\omega_{1}(s)\right)\right)^{\lambda} \nabla s\right)^{\frac{1}{\lambda}} \\
\quad \times\left(\int_{\vartheta_{0}}^{y}(y-\rho(\vartheta))\left(l(\vartheta) \Psi\left(\omega_{2}(\vartheta)\right)\right)^{\mu} \nabla \vartheta\right)^{\frac{1}{\mu}}
\end{gathered}
$$

which is (61). 
Corollary 3.4 If we take $1 / \lambda+1 / \mu=1$ in (61), then

$$
\begin{gathered}
\int_{\vartheta_{0}}^{x} \int_{\vartheta_{0}}^{y} \frac{K(s) L(\vartheta) \Phi(\Pi(s)) \Psi(\Omega(\vartheta)}{\mu\left(s-\vartheta_{0}\right)^{\lambda-1}+\lambda\left(\vartheta-\vartheta_{0}\right)^{\mu-1}} \nabla s \nabla \vartheta \\
\leq W^{*}(\lambda, \mu, x, y)\left(\int_{\vartheta_{0}}^{x}(x-\rho(s))\left(k(s) \Phi\left(\omega_{1}(s)\right)\right)^{\lambda} \nabla s\right)^{\frac{1}{\lambda}} \\
\quad \times\left(\int_{\vartheta_{0}}^{y}(y-\rho(\vartheta))\left(l(\vartheta) \Psi\left(\omega_{2}(\vartheta)\right)\right)^{\mu} \nabla \vartheta\right)^{\frac{1}{\mu}},
\end{gathered}
$$

where

$$
W^{*}(\lambda, \mu, x, y)=\frac{1}{\lambda \mu}\left(x-\vartheta_{0}\right)^{\frac{\lambda-1}{\lambda}}\left(y-\vartheta_{0}\right)^{\frac{\mu-1}{\mu}} .
$$

Remark 3.15 As a particular case of Corollary 3.4, if $\lambda=\mu=2$, then we get

$$
\begin{aligned}
\int_{\vartheta_{0}}^{x} \int_{\vartheta_{0}}^{y} \frac{K(s) L(\vartheta) \Phi(\Pi(s)) \Psi(\Omega(\vartheta)}{s+\vartheta-2 \vartheta_{0}} \nabla s \nabla \vartheta \\
\leq \frac{1}{2}\left(\left(x-\vartheta_{0}\right) \int_{\vartheta_{0}}^{x}(x-\rho(s))\left(k(s) \Phi\left(\omega_{1}(s)\right)\right)^{2} \nabla s\right)^{\frac{1}{2}} \\
\quad \times\left(\left(y-\vartheta_{0}\right) \int_{\vartheta_{0}}^{y}(y-\rho(\vartheta))\left(l(\vartheta) \Psi\left(\omega_{2}(\vartheta)\right)\right)^{2} \nabla \vartheta\right)^{\frac{1}{2}},
\end{aligned}
$$

which is [33, Theorem 3.7].

Remark 3.16 As a particular case of Theorem 3.14, if $\mathbb{T}=\mathbb{Z}, \vartheta_{0}=0$, then $\rho(u)=u-1$ and (61) reduces to

$$
\begin{aligned}
\sum_{s=1}^{p} \sum_{\vartheta=1}^{q} \frac{K_{s} L_{\vartheta} \Phi\left(\Pi_{s}\right) \Psi\left(\Omega_{\vartheta}\right)}{\mu s^{\frac{(\lambda-1)(\lambda+\mu)}{\lambda \mu}}+\lambda \vartheta \frac{(\mu-1)(\lambda+\mu)}{\lambda \mu}} \leq & W_{0}(\lambda, \mu, p, q)\left(\sum_{s=1}^{p}(p-s+1)\left(k_{s} \Phi\left(\omega_{s}\right)\right)^{\lambda}\right)^{\frac{1}{\lambda}} \\
& \times\left(\sum_{\vartheta=1}^{q}(q-\vartheta+1)\left(l_{\vartheta} \Phi\left(\omega_{\vartheta}\right)\right)^{\mu}\right)^{\frac{1}{\mu}},
\end{aligned}
$$

where

$$
W_{0}(\lambda, \mu, p, q)=\frac{1}{\lambda+\mu} p^{\frac{\lambda-1}{\lambda}} q^{\frac{\mu-1}{\mu}},
$$

which is [13, Theorem 2.4].

Remark 3.17 As a particular case of Theorem 3.14, if $\mathbb{T}=\mathbb{R}, t_{0}=0$, then $\rho(u)=u$ and (61) reduces to

$$
\begin{aligned}
\int_{0}^{x} \int_{0}^{y} \frac{K(s) L(\vartheta) \Phi(\Pi(s)) \Psi(\Omega(\vartheta))}{\mu s^{\frac{(\lambda-1)(\lambda+\mu)}{\lambda \mu}}+\lambda \vartheta^{\frac{(\mu-1)(\lambda+\mu)}{\lambda \mu}}} d s d \vartheta \\
\leq W_{0}^{*}(\lambda, \mu, x, y)\left(\int_{0}^{x}(x-s)\left(k(s) \Phi\left(\omega_{1}(s)\right)\right)^{\lambda} d s\right)^{\frac{1}{\lambda}} \\
\quad \times\left(\int_{0}^{y}(y-\vartheta)\left(l(\vartheta) \Psi\left(\omega_{2}(\vartheta)\right)\right)^{\mu} d \vartheta\right)^{\frac{1}{\mu}}
\end{aligned}
$$


where

$$
W_{0}^{*}(\lambda, \mu, x, y)=\frac{1}{\lambda+\mu} x^{\frac{\lambda-1}{\lambda}} y^{\frac{\mu-1}{\mu}},
$$

which is [13, Theorem 3.4].

Remark 3.18 Clearly, Theorems 3.1, 3.6, 3.10, and 3.14 present the corresponding results of Theorems 6, 9, 12, and 15 in [18], respectively, for time scale delta calculus. Likewise, Corollaries 3.1, 3.2, 3.3, and 3.4 display the corresponding results of Theorems 3.1, 3.2, 3.3 , and 3.4 in [17], respectively, for delta time scale calculus.

\section{Acknowledgements}

This research was funded by the Deanship of Scientific Research at Princess Nourah Bint Abdulrahman University through the Fast-track Research Funding Program.

\section{Funding}

This research was funded by the Deanship of Scientific Research at Princess Nourah Bint Abdulrahman University through the Fast-track Research Funding Program.

\section{Availability of data and materials}

Not applicable.

\section{Competing interests}

The authors declare that they have no competing interests.

\section{Authors' contributions}

All authors conceived the study, participated in its design and coordination, drafted the manuscript, participated in the sequence alignment, and read and approved the final manuscript.

\section{Author details}

${ }^{1}$ Department of Mathematics, Faculty of Science, Al-Azhar University, Nasr City 11884, Cairo, Egypt. ${ }^{2}$ Department of Mathematical Science, College of Science, Princess Nourah bint Abdulrahman University, P.O. Box 105862, Riyadh, 11656 , Saudi Arabia. ${ }^{3}$ Department of Mathematics and Computer Science, Faculty of Science, Beni-Suef University, Beni-Suef, Egypt. ${ }^{4}$ Department of Physics, College of Sciences, University of Bisha, P.O. Box 344, Bisha 61922, Saudi Arabia. ${ }^{5}$ Physics Department, Faculty of Science, Al-Azhar University, Assiut 71524, Egypt. ${ }^{6}$ Department of Mathematics, College of Arts and Science, Prince Sattam bin Abdulaziz University, Wadi Aldawaser, Saudi Arabia. ${ }^{7}$ Department of Mathematics, College of Science, King Khalid University, P.O. Box 9004, Z 61413, Abha, Saudi Arabia. ${ }^{8}$ Department of Mathematics, Faculty of Science, Al-Azhar University, Assiut 71524, Egypt.

\section{Publisher's Note}

Springer Nature remains neutral with regard to jurisdictional claims in published maps and institutional affiliations.

Received: 6 July 2020 Accepted: 22 October 2020 Published online: 04 November 2020

\section{References}

1. Hardy, G.H., Littlewood, J.E., Pólya, G.: Inequalities, 2nd edn. Cambridge University Press, Cambridge (1934)

2. Gao, M., Yang, B.: On the extended Hilbert's inequality. Proc. Am. Math. Soc. 126, 751-759 (1998)

3. Jichang, K.: On new extensions of Hilbert's integral inequality. J. Math. Anal. Appl. 235, 608-614 (1999)

4. Handley, G.D., Koliha, J.J., Pečarić, J.E.: New Hilbert-Pachpatte type integral inequalities. J. Math. Anal. Appl. 257, $238-250$ (2001)

5. Yang, B.: On new generalizations of Hilbert's inequality. J. Math. Anal. Appl. 248, 29-40 (2000)

6. AlNemer, G., Zakarya, M., Abd El-Hamid, H.A., Kenawy, M.R., Rezk, H.M.: Dynamic Hardy-type inequalities with non-conjugate parameters. Alex. Eng. J., 1-10 (2020)

7. Abdel-Aty, A., Khater, M.M.A., Attia, R.A.M., Abdel-Aty, M., Eleuch, H.: On the new explicit solutions of the fractional nonlinear space-time nuclear model. Fractals 28, 2040035 (2020)

8. Ereu, J., Gimenez, J., Perez, L.: On solutions of nonlinear integral equations in the space of functions of shiba-bounded variation. Appl. Math. Inf. Sci. 14, 393-404 (2020)

9. Nchama, G.A.M., Mecias, A.L., Richard, M.R.: The Caputo-Fabrizio fractional integral to generate some new inequalities. Inf. Sci. Lett. 8, 73-80 (2019)

10. Abu-Donia, H.M., Atia, H.A., Khater, O.M.A.: Some fixed-point theorems in fuzzy 2-metric spaces under $\psi$-contractive mappings. Inf. Sci. Lett. 9, 21-25 (2020)

11. Pachpatte, B.G.: On some new inequalities similar to Hilbert's inequality. J. Math. Anal. Appl. 226, 166-179 (1998)

12. Kim, Y.-H.: An improvement of some inequalities similar to Hilbert's inequality. Int. J. Math. Math. Sci. 28(4), 211-221 (2001) 
13. Yang, W.: Some new Hilbert-Pachpatte's inequalities. J. Inequal. Pure Appl. Math. 10, 1-14 (2009)

14. Agarwal, R.P., Bohner, M., Peterson, A.: Inequalities on time scales: a survey. Math. Inequal. Appl. 4, 535-557 (2001)

15. Saker, S.: Dynamic inequalities on time scales: a survey. J. Fract. Calc. Appl. 3 (S) (2), 1-36 (2012)

16. Agarwal, R.P., O'Regan, D., Saker, S.: Dynamic Inequalities on Time Scales. Springer, Berlin (2014)

17. Saker, S., Ahmed, A.M., Rezk, H.M., O'Regan, D., Agarwal, R.P.: New Hilbert's dynamic inequalities on time scales. J. Inequal. Pure Appl. Math. 20(40), 1017-1039 (2017)

18. Ahmed, A.M., AlNemer, G., Zakarya, M., Rezk, H.M.: Some dynamic inequalities of Hilbert's type. J. Funct. Spaces 2020, $1-13(2020)$

19. O'Regan, D., Rezk, H.M., Saker, S.: Some dynamic inequalities involving Hilbert and Hardy-Hilbert operators with kernels. Results Math. 73(146), 1-22 (2018)

20. Saker, S., Rezk, H.M., O'Regan, D., Agarwal, R.P.: A variety of inverse Hilbert type inequality on time scales. Dyn. Contin. Discrete Impuls. Syst., Ser. A Math. Anal. 24, 347-373 (2017)

21. Saker, S., Rezk, H.M., Krnić, M.: More accurate dynamic Hardy-type inequalities obtained via superquadraticity. Rev. R. Acad. Cienc. Exactas Fís. Nat., Ser. A Mat. 113, 2691-2713 (2019)

22. Saker, S., Rezk, H.M., Abohela, I., Baleanu, D.: Refinement multidimensional dynamic inequalities with general kernels and measures. J. Inequal. Appl. 2019, 306 (2019)

23. Saker, S., Kenawy, M., AlNemer, G., Zakarya, M.: Some fractional dynamic inequalities of Hardy's type via conformable calculus. Mathematica 8, 434 (2020)

24. Li Nian, W.: Bounds for certain new integral inequalities on time scales. Adv. Differ. Equ. 2009, 484185 (2009)

25. Li Nian, W.: Nonlinear integral inequalities in two independent variables on time scales. Adv. Differ. Equ. 2011, 283926 (2011)

26. Sarfaraz, S., Ahmad, N., Rahman, G.: Study of nonlinear Pachpatte's inequalities on time scales. Adv. Differ. Equ. 2019, 402 (2019)

27. Agarwal, R.P., Hyder, A., Zakarya, M.: Well-posedness of stochastic modified Kawahara equation. Adv. Differ. Equ. 2020 18 (2020)

28. Abd El-Hamid, H.A., Rezk, H.M., Ahmed, A.M., AlNemer, G., Zakarya, M., El Saify, H.A.: Dynamic inequalities in quotients with general kernels and measures. J. Funct. Spaces 2020, 1-12 (2020)

29. AlNemer, G., Zakarya, M., Abd El-Hamid, H.A., Agarwal, P., Rezk, H.M.: Some dynamic Hilbert-type inequalities on time scales. Symmetry 12(9), 1410 (2020)

30. Bohner, M., Peterson, A.: Dynamic Equations on Time Scales: An Introduction with Applications. Birkhäuser, Boston (2001)

31. Bohner, M., Peterson, A.: Advances in Dynamic Equations on Time Scales. Birkhäuser, Boston (2003)

32. Özkan, U.M., Sarikaya, M.Z., Yildirim, H.: Extensions of certain integral inequalities on time scales. Appl. Math. Lett. 21, 993-1000 (2008)

33. Anderson, D.R.: Dynamic double integral inequalities in two independent variables on time scales. J. Math. Inequal. 2 163-184 (2008)

34. Mitrinovic, D.S., Pecaric, J.E., Fink, A.M.: Classical and New Inequalities in Analysis. Kluwer Academic, Dordrecht (1993)

\section{Submit your manuscript to a SpringerOpen ${ }^{\circ}$ journal and benefit from:}

- Convenient online submission

- Rigorous peer review

- Open access: articles freely available online

- High visibility within the field

- Retaining the copyright to your article

Submit your next manuscript at $>$ springeropen.com 